\title{
GCU
}

Glasgow Caledonian

University

University for the Common Good

\section{Acceptability and feasibility of peer assisted supervision and support for intervention practitioners: a Q-methodology evaluation}

McPherson, Kerri; Sanders, Matthew; Schroeter, Birgit; Troy, Victoria Jayne; Wiseman, Kirsty

Published in:

Journal of Child and Family Studies

DOI:

10.1007/s10826-015-0281-9

Publication date:

2016

Document Version

Author accepted manuscript

Link to publication in ResearchOnline

Citation for published version (Harvard):

McPherson, K, Sanders, M, Schroeter, B, Troy, VJ \& Wiseman, K 2016, 'Acceptability and feasibility of peer assisted supervision and support for intervention practitioners: a Q-methodology evaluation', Journal of Child and Family Studies, vol. 25, no. 3, pp. 720-732. https://doi.org/10.1007/s10826-015-0281-9

\section{General rights}

Copyright and moral rights for the publications made accessible in the public portal are retained by the authors and/or other copyright owners and it is a condition of accessing publications that users recognise and abide by the legal requirements associated with these rights.

Take down policy

If you believe that this document breaches copyright please view our takedown policy at https://edshare.gcu.ac.uk/id/eprint/5179 for details

of how to contact us. 


\begin{abstract}
Evidence-based interventions often include quality improvement methods to support fidelity and improve client outcomes. Clinical supervision is promoted as an effective way of developing practitioner confidence and competence in delivery; however, supervision is often inconsistent and embedded in hierarchical line management structures that may limit the opportunity for reflective learning. The Peer Assisted Supervision and Support (PASS) supervision model uses peer relationships to promote the self-regulatory capacity of practitioners to improve intervention delivery. The aim of the present study was to assess the acceptability and feasibility of PASS amongst parenting intervention practitioners. A Q-methodology approach was used to generate data and 30 practitioners volunteered to participate in the study. Data were analyzed and interpreted using standard Q-methodology procedures and by-person factor analysis yielded three factors. There was consensus that PASS was acceptable. Participants shared the view that PASS facilitated an environment of support where negative aspects of interpersonal relationships that might develop in supervision were not evident. Two factors represented the viewpoint that PASS was also a feasible model of supervision. However, the third factor was comprised of practitioners who reported that PASS could be time consuming and difficult to fit into existing work demands. There were differences across the three factors in the extent to which practitioners considered PASS impacted on their intervention delivery. The findings highlight the importance of organizational mechanisms that support practitioner engagement in supervision.
\end{abstract}

Keywords: Q-methodology, supervision, parenting interventions, evaluation, program implementation 


\section{Introduction}

Evidence-based parenting and family support (PFS) interventions often include quality improvement methods that are generally accepted in the intervention science literature as supporting program fidelity and improved client outcomes ( Henggeler, 2011; Sanders, Prinz, \& Shapiro, 2009). However, it can be difficult to translate the implementation and monitoring of these methods from the highly controlled efficacy environment to a service delivery setting and, consequently, many delivering organizations view them as undesirable or unsupportable (Fixsen, Naoom, Blasé, Friedman \& Wallace, 2005; Henggeler, 2011; Schoenwald, Sheidow, \& Chapman, 2009). They may be perceived as incompatible with existing organizational structures and culture, or as a costly accessory to the PFS intervention itself. Clinical supervision, or mentoring and coaching, of the workforce delivering PFS interventions is one such quality improvement method.

A range of definitions of clinical supervision have been offered and, while acknowledging nuanced differences between these, there is general acceptance that clinical supervision is a "formal process of professional support and learning which enables practitioners to develop knowledge and competence, assume responsibility for their own practice and enhance consumer protection and the safety of care in complex clinical situations." (Department of Health.National Health Service Management Executive, 1993). It is assumed that clinical supervision supports the development of practitioners' confidence and competence and, in turn, supports them achieving the best client outcomes (Fixsen, Blase, Duda, Naoom, \& Van Dyke, 2010; Sanders et al., 2009). For example, in one of the few reviews of the supervision literature, there was evidence to suggest that supervision enhances practitioners' self-efficacy, skills and self-awareness (Wheeler \& Richards, 2007).

While clinical supervision is well embedded in some professions, such as counseling and clinical psychology (Davy, 2002), it is not a standard requirement for the majority. There is considerable disparity in the models employed, the value it is given, and the relationship it shares with line management is variable. These differences may contribute to disparity in the organizational sustainability of supervision (Davy, 2002; Wheeler \& Richards, 2007). This is amplified in the case of PFS interventions that have been designed to be delivered by practitioners from different professional backgrounds (e.g., Triple P-Positive Parenting Program and Incredible Years), including allied health, education, social welfare and not for profit sector backgrounds. While these practitioners may appear to be an interdisciplinary workforce, they are normally retained within existing organizations or teams 
and thus constrained by existing structures for supervision and management. This makes it difficult to ensure consistency in the implementation of the supervision requirements of the PFS intervention and thus potentially compromises the quality assurance structures of the overall program.

There have been repeated calls for research to test the hypothesized link between quality improvement methods, such as clinical supervision, and client outcomes, but the evidence is limited (Henggeler, 2011; Schoenwald et al., 2009). Schoenwald et al. (Schoenwald, Sheidow, \& Letourneau, 2004; Schoenwald et al., 2009) were among the first to demonstrate the link between practitioner supervision and child outcomes in a child-focused treatment intervention. In addition, practitioners trained in a prevention intervention noted lack of supervision as a key barrier to program implementation (Sanders et al., 2009). On the other hand, the opportunity to consult with colleagues trained in the same intervention was noted as a facilitator of intervention use (Sanders et al., 2009). While these results are promising, the paucity of evidence showing a link between clinical supervision and client outcomes may act as a barrier to its rigorous adoption in a service context (Berggren et al., 2005; Schoenwald et al., 2009; Tony, Louise, Christine, \& Majda, 2008).

An alternative approach to clinical supervision for PFS intervention practitioners is a systemscontextual one that is responsive to dynamic organizational and individual factors that influence implementation (Sanders \& Murphy-Brennan, 2010). Specifically, any intervention-related supervision model needs to be sensitive to existing organizational supervision and management arrangements, rather than seeking to circumvent these or to add to the administrative burden of practitioners. In addition, and of particular importance in the case of PFS interventions, the model needs to demonstrate the flexibility to be implemented consistently across an interdisciplinary workforce. Changing the agent of supervision from the, often hierarchical, supervisor to the practitioners themselves is one way in which this might be achieved.

There is extensive literature on the positive role that peers can have in a large number of domains. For example, a recent systematic review found that peers can be used to achieve client outcomes that are similar to those produced by professionals in mental health services (Pitt et al., 2013). Others have written about the benefits of group supervision for psychotherapists and highlight the potential for the group to: generate a wider range of solutions to problems encountered in clinical practice than one-to-one supervision; reduce professional isolation; develop professional identity and esteem; and, reduce the workforce cost associated with supervision (Counselman \& Weber, 2004). 
Acknowledging this, the Peer Assisted Supervision and Support (PASS) model (Sanders \& Murphy-Brennan, 2013) has been developed as a model of supervision that seeks to promote the self-regulatory capacity of practitioners by capturing the empowering nature of peer relationships. PASS evolved into a manualized quality enhancement model of supervision for parenting intervention practitioners from experience gained in implementing the Triple P-Positive Parenting Program in a range of different settings (Sanders, 2008; Sanders, 2012). As with other evidence-based interventions, although practitioners received systematic training in the parenting intervention, when they began delivering Triple $\mathrm{P}$ a range of practical issues arose that they sought support, guidance and reassurance about. Peer practitioners became a natural and valuable source of support. While the PASS model was developed in response to the needs to of practitioners delivering Triple $\mathrm{P}$, and is now embedded in the Triple P Implementation Framework (McWilliam, Brown, Sanders \& Jones, 2015), the focus on developing self-regulatory capacity using peer relationships means it is independent of the content of the PFS intervention and its framework for supervision could be applied to support the delivery of other interventions.

The model provides instruction on self-reflective practice and is designed to facilitate personal confidence, learning and decision making (Mazzucchelli \& Sanders, 2010). It uses peer relationships as a supportive and motivational scaffold for the development of practitioners' self-regulatory skills, which enables the appropriate modification of each practitioner's own behavior in a goal directed way while taking account of changing circumstances (Karoly, 1993). In the context of PFS intervention delivery, these skills include how to: select developmentally appropriate goals; choose an appropriate method of intervention to address a particular problem; implement the solution and self-monitor this via checklists relating to the areas of concern; and, identify strengths or limitations in performance and set future goals for action (Sanders, 1999). The self-regulation approach to supervision aims to empower practitioners to make the changes they deem necessary to successfully implement an intervention with clients. It encourages peers to assess and share observations about the competencies and challenges faced by other practitioners, and it provides a motivational context to enable the practitioner to change their own behaviors, cognitions and emotions so they become more proficient in intervention delivery. While proposed as an alternative to traditional hierarchical supervision, the model can be implemented flexibly as one to complement or replace existing organizational structures and processes. 
Guided by a manual (Sanders \& Murphy-Brennan, 2013) and demonstration DVD, small groups of practitioners $(n=4-6)$ meet regularly, normally every two weeks, rotating the roles of peer facilitator, peer mentor and practitioner. PASS session focus on three key activities: case review of delivered intervention sessions; discussion of implementation issues; and, future actions goal setting and professional development. When acting as peer facilitator, the practitioner takes responsibility for setting up and facilitating the PASS session. The case review element of the session is normally managed by the practitioners bringing with them a short (5-10 minute) recorded segment of an intervention session that they want to discuss and receive feedback on. The peer mentors offer feedback on the cases being reviewed and prompt self-evaluation from the practitioner presenting the case. By rotating the roles within the peer group individual practitioners are afforded the opportunity to give and receive feedback in a safe but challenging environment. Moreover, each individual learns by reflecting on the cases presented by their peers.

Given the lack of evidence about the impact of supervision on practitioners' delivery and intervention outcomes, the aim of the present study was to assess the acceptability and feasibility of the PASS model of supervision amongst parenting intervention practitioners and generate data to underpin the development of future randomized controlled trials. This phase is recognized as a key stage in the evaluation of complex interventions in health care settings (Craig et al., 2013). It provides opportunity to refine the intervention materials and procedures, and to better understand the individual and organizational complexities and constraints associated with implementation prior to full scale evaluation (Ayala \& Elder, 2011; Craig et al., 2013; Steckler \& Linnan, 2003). This is further enhanced by employing an approach that explores the subjective views of key stakeholders and, therefore, enables consideration of the elements of the intervention that are more or less acceptable, for whom and in what context.

Much of the research designed to assess subjective dimensions of intervention acceptability and feasibility has employed traditional interview and focus group methods to generate qualitative data and, in some cases, attempts are made to triangulate this with quantitative data (Ayala \& Elder, 2011; Breitenstein \& Gross, 2013; Haines et al., 2012). An alternative approach lies in the use of Qmethodology, a technique designed to combine elements of qualitative and quantitative research methods to facilitate the measurement of subjectivity (Baker, Thompson, \& Mannion, 2006; Stephenson, 1953). Like qualitative methods, Q-methodology seeks to identify patterns of diversity 
and similarity in the viewpoints of individuals and it is primarily used to assess personal experiences, opinions and beliefs about the topic of interest (McKeown \& Thomas, 1988). However, the Qmethodology approach to data collection and analysis is more akin to quantitative techniques. Specifically, elements of subjectivity are accessed by engaging study participants in a Q-sort task where each individual ranks a set of statements about the topic of interest on a scale indicating their level of agreement or disagreement with each one (Watts \& Stenner, 2012). The end products of the task, the individual Q-sorts, are then subject to by-person factor analysis to establish patterns of convergence and divergence across the viewpoints of each individual (Watts \& Stenner, 2012).

Thus, as noted by Baker et al. (Baker et al., 2006) in relation to health economics, one of the key strengths of $Q$ is its ability to address research questions more aligned with qualitative paradigms using quantitative principles. This makes it intuitively appealing in the context of intervention research which relies on transparency in the analytic process. In addition, while traditional qualitative methods enable the generation of data about the acceptability and feasibility of interventions, the statistical grouping of participants based on patterns of shared agreement and disagreement affords intervention researchers greater opportunity to explore factors that might underpin participants' subjective views (Sexton, Snyder, Wadsworth, Jardine, \& James, 1998). Thus the current study employed a Q-methodology approach to assess the acceptability and feasibility of the PASS model of supervision amongst parenting intervention practitioners.

\section{Method}

\section{Participants}

Participants were recruited by seeking volunteers from a pool of 36 practitioners who had been trained to deliver a parenting intervention as part of a Scottish Government early intervention workforce development initiative. By way of context, the Psychology of Parenting Project (PoPP) was delivered through NHS Education Scotland and focused on capacity building around evidence-based parenting programs targeting 3-4 year old children with elevated levels of behavior problems.

Practitioners received one day per week protected work time to deliver the parenting intervention and participate in PASS.

As part of the parenting intervention training, participants were introduced to the PASS model of supervision and they received the PASS manual. This training took place eight months prior to the 
Q-methodology data collection. Following training, and as part of their professional development, practitioners participated in complementary learning facilitated by the PoPP team, about: the development of behavioral difficulties; fidelity monitoring and its role in evidence based interventions; strengths-based communications to promote parental engagement; and, working within a data driven decision making system. In the early phase of implementation, the PoPP team supported the PASS groups through regular visits. These visits included: prompting practitioners to adhere to the roles defined in the PASS model; prompting to use the self-regulatory framework that underpins the PASS model; and, if necessary, modeling how to give and receive constructive feedback.

The 36 potential participants were sent a leaflet that gave information about the nature and purpose of the Q-methodology study and invited them to attend a data collection session. A total of 30 individuals volunteered to participate in the Q-sort task. Six participants submitted incomplete Q-sorts and they were excluded from further analysis, this left a final sample of 24 participants or 24 analyzable Q-sorts.

\section{Procedure}

The success of any Q-study rests in the development of a diverse set of materials (Q-items) that can be used to assess the views, perceptions and experiences of participants. In the current study this meant the generation of items, or statements, that could capture participants' views, perceptions and experiences of the PASS intervention. As a first step, the research question to be presented to participants as their guide for the task was defined: "As a [parenting intervention] practitioner, what are your experiences of participating in PASS supervision?"

Next, using three different sources of information, initial Q-items were developed by three members of the research team (KEM, BS and KW) working independently from the remaining members of the team. Items were generated from: 1) published literature about the nature and purpose of supervision (Counselman \& Weber, 2004; Davy, 2002; Turpin \& Wheeler, 2011; Wheeler \& Richards, 2007) and published research about people's experiences of participating in supervision (Cheater \& Hale, 2001); 2) information published in the PASS manual about the nature and purpose of the PASS model of supervision ( Sanders \& Murphy-Brennan, 2013); and, 3) transcripts from focus groups with practitioners who had previously participated in PASS (Ward, 2013). This resulted in a heterogeneous set of 122 Q-items. Where possible, each item was phrased around a first person 
pronoun to ensure that participants were reflecting on their own personal experiences of participating in PASS.

Next, the 122 Q-items were reviewed by two colleagues with expertise in supervision in the context of parenting interventions; they were asked to refine the Q-items to a meaningful and manageable Q-set. They assessed each item for understanding and clarity of meaning; for example, items expressed as double negatives and those with dual interpretation were rejected. In tandem, they assessed the holistic set of items to ensure that duplication was avoided, diversity was enhanced, and each item was relevant in the context of the research question to be posed to participants. The final Q-set was comprised of 64 items (available on request from the authors).

The sorting of the 64 item Q-set was carried out by participants in two groups that were constituted on the basis of location convenience. Each group was given a short demonstration of the sorting procedure using an unrelated example. Participants were seated at a desk that had a copy of the research question placed at the top of it; it read "As a [parenting intervention] practitioner, what are your experiences of participating in PASS supervision?" It was explained that they should read each of the items in the Q-set, sorting them into three piles: items they agreed with, items they disagreed with, and items they had no strong feelings about.

Once they had completed this, participants were asked to arrange the items on a large Q-sort grid that had been placed on their desk. The $Q$-sorting grid had a response scale that ran from +5 'strongly agree' to -5 'strongly disagree'. Participants were asked to sort each item into one of the blank cells on the grid based on the strength of their feeling about it. They were asked to complete the task by starting with the items in their 'agree' pile before moving onto their 'disagree' pile and finally the pile of items that they had no strong feeling about.

The grid in this study had a forced choice distribution meaning that participants could only place items into cells provided on the grid and each cell could only contain one item (i.e. they could not change the shape of the distribution). Participants were encouraged to rearrange the items on the grid until they were satisfied with the item placement, at which point they were asked to populate a paper copy of the grid using the unique item number on each item card.

Finally, following standard Q-methodology practice (Watts \& Stenner, 2012), participants were asked to write a short explanation of what the items that they strongly agreed with, and strongly disagreed with, meant to them. They were also offered the opportunity to provide comment on their 
experience of PASS if they felt the Q-items had not included an important element of their experience. Participants were also asked their sex, their current profession and their previous experience of supervision.

\section{Data Analyses}

Data was analyzed using PQ Method 2.35 software (Schmolck, n.d.) to conduct a centroid factor analysis. The focus of the analysis were the Q-sorts produced by each participant and the analysis was conducted by-person rather than on the individual items (Watts \& Stenner, 2012). The correlation matrix reflects the relationships between each of the Q-sorts (i.e. each participant), and the factors produced in the analysis are comprised of Q-sorts that are similar in the placement of items (Watts \& Stenner, 2012). Thus, the factors represent different views about the topic of interest, one that is shared by the participants (Q-sets) that load on that factor. The qualitative comments provided by participants about their placement of Q-items and additional comments about the PASS experience were used to facilitate the interpretation of the factors and illustrative examples are presented in the results section.

\section{Results}

\section{Initial Analysis: Determining the Factors}

The unrotated solution from the factor analysis was inspected to determine the number of factors to retain. Only factors that had two or more Q-sorts loading significantly on them $(\geq 0.50)$ were retained (Brown, 1980). This, coupled with inspection of the eigenvalue scree plot, lead to three factors being retained. The solution was rotated orthogonally using a varimax method and the three factors accounted for $57 \%(F 1=19 \%, F 2=12 \%, F 3=25 \%)$ of the total variance.

A number of different criteria exist for determining which Q-sorts are considered to exemplify a factor (Watts \& Stenner, 2012). In this study a Q-set was considered to exemplify a factor if it loaded at $\geq 0.5$ on the factor and its loadings on other all factors were at least 0.1 (rounded to one decimal place) less than this. Q-sets where the difference between the two highest factor loadings was $<0.1$ were considered to be confounded and excluded from factor estimates. Of the 24 usable Q-sets, 19 were identified as exemplifying one of the three factors, three were confounded and two did not meet the criteria to be considered as loading significantly on one of the three factors. Table 1 shows the factor matrix with the exemplifying Q-sorts highlighted in bold. 
INSERT TABLE 1 ABOUT HERE

\section{Interpreting the Factors}

\section{Consensus across the factors.}

While much of the interpretation of Q-data is normally focused on the differences that exist between factors and the viewpoints they represent, understanding where there is consensus across participants is important in the context of establishing the acceptability and feasibility of an intervention such as PASS. It provides insight into what is and is not acceptable and feasible for the majority. The 15 statements where there was consensus among any pair of the three factors extracted in this study are displayed in Table 2.

\section{INSERT TABLE 2 ABOUT HERE}

There was agreement across the three factors that having supervision with colleagues from different professional backgrounds was enjoyable (1) and that PASS was a supportive experience (3). In contrast, there was consistent disagreement that attending PASS sessions induces nervousness (4) or that bringing a problem to a PASS group was a fearful experience (18). The Q-data was complemented by comments provided by participants.

Our PASS group are all very supportive to each other, everyone feels open and honest to talk freely. (Participant L)

There was agreement across the three factors that in PASS sessions practitioners found it easy to describe a problem that they would like feedback on (14), and having sought this feedback they could see what they would need to change (15). Practitioners, particularly those in Factors 1 and 3 , disagreed that they were unsure how to put the feedback from peers into action (63). This suggests that practitioners represented by all three factors felt at ease with the processes of eliciting feedback in their groups. 
To a lesser extent, there was agreement across the three factors that rotating PASS roles was useful (10). Disagreement on all three factors that the success of PASS sessions rests too heavily on the shoulders of the facilitator (24) was coupled with disagreement on two factors (factors 1

and 3 ) that the administrative expectations of the facilitator role was too time consuming (22). In addition, practitioners across the three factors disagreed that the video footage presented to facilitate feedback in the PASS sessions got in the way of discussions (64). Thus, many of the key processes in PASS (rotation of roles, giving and receiving feedback, use of video) appeared to be acceptable to practitioners.

In what follows, the interpretation of each of the three extracted factors is described. The interpretation is based on a holistic analysis of: the items rated at the extreme end of the rating scale ( \pm 5 and \pm 4 ), described by Baker (Baker et al., 2006) as a factor's 'salient items'; the factor scores; the items identified by PQMethod as distinguishing each factor from the other two; and, the qualitative comments provided by the participants.

\section{Factor 1.}

Six practitioners loaded on Factor 1, which accounted for $19 \%$ of the total variance, the salient items for this factor are displayed in Table 3. There were three females and two males from a range of professional backgrounds; including educational psychology, social work and family support.

\section{INSERT TABLE 3 ABOUT HERE}

Factor 1 represents a shared view that defines participation in PASS as a supportive experience (3) where there was no need for nervousness (4). There was a rejection of the idea that moving to a new PASS group would raise anxiety (8) and that PASS groups can only work if they were given time to bond (7). This suggests that these practitioners view the supportive element of PASS as being more than just interpersonal support achieved through discussion with familiar colleagues. They articulated an ease with the processes outlined in the PASS manual (12), which emphasizes the need to elevate the group above social support to focus on developing constructive peer feedback mechanisms and the enhancement self-regulatory skills.

Indeed, the pattern of item endorsement by these practitioners suggests that they valued the peer feedback that is embedded as the key process in the PASS model. They described being 
confident asking for feedback from their peers (13) and, in return, comfortable giving positive feedback to their peers (25).

I...strongly agree about feeling confident about asking my peers for feedback on how I delivered [the] parenting intervention. This is one of the great strengths of PASS that it allows us to do this in a comfortable environment. (Participant C)

Moreover, the practitioners were comfortable providing peers with feedback and suggestions on what they might have done differently (26) (i.e., constructive negative feedback) and disagreed that they found receiving negative feedback difficult to handle (17). They reported confidence taking on the role of practitioner in PASS sessions (19); this is the role where practitioners offer segments of their intervention delivery for the group to discuss. They also rejected the need for an expert to lead the groups (11) and rejected the notion that peers were too inexperienced to provide useful feedback (16).

While appreciation of bidirectional peer feedback is an important element in the development of self-regulatory capabilities, there was limited evidence from this group of practitioners that they used the peer feedback to enact positive and goal directed change in their delivery of the parenting intervention. They had fairly neutral views about items describing change in their practice $(33,39,44)$. It is not possible to know from the data available if this resulted from an inability to translate feedback into practice or, for example, a confidence that their practice was already appropriate and not in need of change. Their neutral endorsement of PASS's ability to help them develop problem solving skills (35) might support the latter. However, their disagreement with the success of PASS is being contingent on the use of recorded segments of delivery (9) might support the former in that these practitioners may not have been using the recordings as a facilitator of feedback and self-regulatory goal directed change.

The sharing of videos was a barrier to begin with. ICT [information and computer technology] issues training was required. (Participant D) 
Finally, this factor provides some insight into the practitioners' views about the feasibility of PASS in wider work context. Practitioners rejected the view that time spent on PASS would be better spent on other elements of their job (57) and were neutral about it being hard to fit PASS into their working schedule (53). This willingness or ability to incorporate PASS into current work demands is likely to be a result of the perception that they had line manager support (55).

In sum, the salient and defining items for this factor represented a shared view about the value of PASS and in particular the opportunity to give and receive feedback from peers. There was a high degree of acceptability of peer feedback amongst this group of practitioners and a rejection that peers were not expert enough to provide useful feedback. However, their translation of feedback into goal-directed change and enhanced practice is less clear. This factor also described a group of practitioners who felt they had organizational support to participate in PASS and that they could fit it in their workload. In other words, they found PASS both acceptable and feasible but it remains unclear what impact participation in PASS had on their delivery of the parenting intervention.

\section{Factor 2.}

Factor 2 accounted for $12 \%$ of the variance and represented the shared views of four practitioners, the salient items for this factor are displayed in Table 4. One practitioner was male and one was female, the other two practitioners chose not to record their sex.

\section{INSERT TABLE 4 ABOUT HERE}

The shared view of practitioners represented by Factor 2 was, again, one that articulated PASS as a supportive experience (3) where nervousness (4) did not feature. As with Factor 1, many of the salient items focused on feedback. The practitioners felt confident about eliciting feedback from their peers (13) and in the role of practitioner (19) when practitioners offered segments of their intervention delivery for the group to discuss. They were also comfortable giving both positive (25) and constructive negative feedback (26) to peers about their practice.

I do feel very comfortable about giving feedback to my colleagues.

(Participant I) 
However, when viewed holistically, the overall focus of the items that distinguish Factor 2 from the other two factors is on what PASS does not do. Unlike Factor 1 where items about how PASS impacts on delivery of the parenting intervention were not identified as being part of the distinguishing pattern, in Factor 2 they were. This group of practitioners disagreed that PASS made them more confident drawing conclusions about parents' problem(s) (39), that PASS improved the quality of the care they gave families (45), and that PASS helped them see how actions resulted in improvements in families they worked with (41). They were also less inclined than those on Factors 1 and 3 to report that PASS made them more confident to deliver the parenting intervention (44), tailor the intervention for each group of parents (47) and manage fidelity of the delivery (46).

The focus on what PASS did not do for this group of practitioners also translates into the wider work context. For example, the practitioners did not feel PASS helped them reflect on performance at work (49). They disagreed that PASS helped them regulate emotional reactions to work situations (48) and disagreed that it equipped them to manage conflict (51) or stress (50) in the workplace. They also disagreed that it had enhanced their job satisfaction (52).

It may be that the disconnect between practitioners' positive endorsements of the giving and receiving of feedback in PASS sessions and their perceptions that this did not impact on their delivery of the parenting intervention, or other aspects of their work, related to their understandings of the purpose of PASS sessions. Specifically, they showed some agreement, albeit low level agreement, that PASS is not really supervision (60) and this was further elaborated by practitioners disagreeing that PASS helped them develop problem solving skills (35).

PASS sessions may have helped understanding of [parenting intervention] issues in the program but not as a practitioner overall. (Participant H)

As this quote illustrates, this group of practitioners may have valued the opportunity to share factual learning about the specifics of the parenting intervention, through the giving and receiving of feedback, but did not further develop this to support their on-going delivery and other elements of their working lives. While the available data limits full investigation of this, they point to a group of 
practitioners who may have used PASS to build specific knowledge about a new parenting intervention but who felt confident that they already had the professional skills to deliver it and manage other aspect of their work.

In terms of feasibility, the practitioners represented by Factor 2 found it hard to fit PASS into their busy work schedule (53) and felt PASS meetings were frequent enough (56).

With many competing demands on my time committing time to attend is difficult... Added time pressures to attend PASS adds to my work related stress rather than diminishes it ...Weekly sessions I think are too often! (Participant I)

Thus, while the practitioners represented by Factor 2 experienced PASS as supportive and felt confident and comfortable in giving and receiving feedback, they did not appear to find participating in PASS as acceptable as practitioners represented by Factor 1 . They generally disagreed that PASS enhanced their delivery of the parenting intervention and disagreed that it had wider benefits in terms of managing their work. Furthermore, they articulate perceived feasibility issues related to fitting PASS in with other work-related demands.

\section{Factor 3.}

Factor 3 accounted for $25 \%$ of the variance and represented the shared viewpoint of nine practitioners, the salient items for this factor are displayed in Table 5. The majority of the practitioners were female $(n=8)$ and worked as family support workers.

\section{INSERT TABLE 5 ABOUT HERE}

As can be seen in Table 5, the shared view of practitioners represented within this factor is one of PASS being a supportive experience (3), where practitioners didn't get nervous (4) or fearful (18). The potential for one-to-one supervision to be conceptualized as superior to group supervision (62) was rejected, rather practitioners felt benefit from having undertaken supervision with peers from other professional backgrounds $(1,2)$. 
Everyone is doing the same group and experiencing similar achievements and difficulties and the group help each other work through this. We all have different jobs but the main aim is the same for [parenting intervention] delivery. (Participant P)

Despite this, they disagreed that PASS helped balance power relationships in supervision (58).

Like Factors 1 and 2, practitioners represented on Factor 3 described feeling comfortable giving feedback to their peers (25) and confident in asking peers for feedback (13); they rejected the notion that peers are too inexperienced to offer helpful feedback (16).

I think the support of peers who are delivering the group is far more valuable than that of a manager/expert. (Participant S)

This is further developed by their disagreement that PASS groups avoided looking at relevant issues (61). Unlike Factors 1 and 2, participants loading on Factor 3 articulated a direct link between the feedback that they got in their PASS sessions and their subsequent delivery of the parenting intervention.

PASS is important to me and very useful for my confidence that I am delivering my group work effectively. (Participant M)

They disagreed that PASS groups aren't a place to learn new skills (34). These practitioners described PASS as an environment that facilitated self-regulatory processes and they felt they experienced the benefits of participating in PASS when working with families (42).

Looking at what I do well has increased my confidence as a practitioner.

Being able to recognize what I need to change makes me a better practitioner and helps to develop my skills. (Participant O) 
This factor described practitioners who used PASS to identify elements of their practice that needed to be adapted (33) and elements that were working well (32). Moreover, these practitioners recognized the positive impact that this had on the ways they worked with families $(33,40,42)$. Many of the distinguishing items for this factor were about the ways in which participating in PASS enhanced the delivery of the parenting intervention by, for example, developing problem solving skills and confidence (e.g., 32, 35, 38, 40, 43, 44).

Being able to see how I interact with parents through the video clips helps me take note of how I react to questions etc. It has helped me to be more mindful and aware of how my body language comes across to parents. (Participant K)

Some important issues in terms of the feasibility of PASS were highlighted in this factor. Specifically, while they disagreed that it was hard to fit PASS into their working lives (53), the practitioners suggest that organizational infrastructure to support PASS sessions, such as equipment and meeting space, (54) was an important consideration.

My organization [name removed] does not support any form of supervision and it has been a battle to be allowed to go. (Participant R)

Thus practitioners represented by Factor 3 expressed a shared view of PASS as being an acceptable model of supervision. The peer element of this model of supervision was not undervalued and the idea that peers are too inexperienced was rejected. Importantly, these practitioners articulated a view that PASS supported and enhanced their delivery of the parenting intervention by facilitating self-regulatory skills such as problem solving and reflection.

\section{Discussion}

While previous research has demonstrated that supervision can enhance the professional qualities (e.g., self-efficacy) and skills of psychotherapists (Wheeler \& Richards, 2007) and that supervision may enhance client outcomes (Schoenwald, Sheidow \& Chapman, 2009), evidence about 
the acceptability and feasibility of supervision, from the professionals' perspective, is limited. The aim of the current study was to assess the acceptability and feasibility of a new model of supervision, PASS, designed to enhance the quality of parenting and family support intervention delivery.

The consensus across the practitioners represented on each of the three factors in this study was that the PASS model facilitated an environment of support where the negative aspects of interpersonal relationships that might develop in the context of supervision, such as nervousness and fear, were not evident. A safe and cooperative environment was acknowledged as an important foundation for the development of a fruitful supervisory relationship (Watkins \& Scaturo, 2013). Thus, by creating a safe supervisory environment, PASS has more potential to support the development of practitioner skills, including self-regulatory skills, associated with better client outcomes and this hypothesized relationship requires testing in future research.

Linked to this, the consensus statements pointed to practitioners being at ease with the key processes within the PASS model; namely, peer feedback and the allocation and rotation of roles to facilitate this feedback. Peers were also viewed as being an appropriate alternative to expert-led supervision. As noted above, many evidence-based PFS interventions prescribe an intensive supervision program that spans the lifetime of intervention delivery and this may prove challenging for organizations to resource (Henggeler, 2011; Schoenwald, Sheidow, \& Chapman, 2009). However, in addition to being acceptable to supervisees, a model that is built on peer rather than expert and/or line management supervision has the potential for greater organizational flexibility. For example, using peers means there are more supervisors available, and small peer groups may have more flexibility to plan when and where supervision takes place rather than having to rely on limited opportunity to meet with a single expert/line manager supervisor. Indeed, the availability of supervisors/supervisees has proved a significant barrier in other reviews of supervision (Schoenwald, Mehta, Frazier \& Shernoff, 2013).

Practitioners were also positive about supervision that crossed professional boundaries. This interdisciplinary approach is unusual in traditional models of supervision, which tend to be constrained within disciplinary boundaries and hierarchies (Davy, 2002). However, as service delivery models shift to emphasize interdisciplinary team working, flexible models of supervision that transcend professional boundaries, such as PASS, may be better able to support practitioners. Moreover, the focus on self-regulatory skills building, rather than just profession-specific skills, may provide 
practitioners with a portable tool kit that is responsive to changes in the service in which they work. That said, although an interdisciplinary approach may be assessed as positive by practitioners, the logistics of managing this across multiple agencies requires full consideration; specifically, previous research has identified different organizational structures as a barrier to interagency supervision (Schoenwald, Mehta, Frazier \& Shernoff, 2013).

In terms of feasibility, Factors 1 and 3 both represented shared views that PASS can be incorporated into existing work schedules. Factor 2, on the other hand, represented a shared view that participating in PASS was time consuming and difficult to fit in. It may be that when practitioners find it difficult to prioritize PASS over other competing demands they have limited opportunity to fully prepare for and exploit the process in PASS and, therefore, limit opportunity for positive gain. Alternatively, practitioners who do not experience positive gain from engaging in PASS may be more inclined to see it as a time burden. It is not possible with the present data set to disentangle this further, but evidence within the implementation science field highlights a range of factors that might help elucidate this. For example, practitioners represented by Factor 2 may be at an earlier stage of the psychological change process and be struggling to reconcile their previous role with expectations in relation to the delivery of the new PFS intervention (Aarons, 2004). Alternatively, they may be working within an organization that, for multiple reasons (e.g., staff illness), is unable to prioritize supervision of staff (Aarons, Hurlburt \& Horwitz, 2011; Sanders et al., 2009). This highlights the importance of organizational mechanisms that help identify and breakdown barriers to an individual's participation in PASS. For example, creating dedicated work time for practitioners to engage in supervision would facilitate prioritizing.

While the peer feedback element of PASS was consistently endorsed as being acceptable, the three factors differed in the views they held in relation to the ways in which PASS supported delivery of the parenting intervention and other aspects of the practitioners' working lives. The shared viewpoint expressed in Factor 1 did not articulate a link between peer feedback and future practice. That is not to say that participation in PASS did not result in positive benefits in practitioners' delivery of the parenting intervention, but rather they didn't explicitly express this. In contrast, practitioners represented by Factor 2 made explicit their view that participation in PASS did not result in positive benefits in the way they delivered the parenting intervention, and practitioners represented by Factor 3 made explicit their view that participation in PASS did result in positive benefits in their delivery of 
the intervention. Importantly, while a limited body of research has been able to draw a link between supervision and practitioner delivery (e.g., enhanced fidelity), and in some instances client outcomes, there is no available evidence describing the relationship between practitioners' views about supervision and their subsequent intervention delivery (Schoenwald et al., 2009; Wheeler \& Richards, 2007). When taking on the call to use experimental research to test the hypothesized relationship between supervision and client outcomes (Henggeler, 2011; Schoenwald et al., 2009), future research must also test the relationship between practitioner expectancies about supervision and their practice and client outcomes. Only then will it be possible to determine if practitioners' views about the value, or otherwise, of supervision in relation to practice is borne out in the quality of the service they offer.

\section{Strengthens and Limitations}

One of the major strengths of this study was the adoption of a Q-methodology approach, which allowed for the systematic investigation of practitioners subjective views about participating in PASS. While other approaches to assessing acceptability have been advocated (Ayala \& Elder, 2011), Q-methodology has been demonstrated to be an attractive and robust alternative (Baker et al., 2006; Cross, 2005; Stephenson, 1953). That said, Q is not without its limitations. In particular, the development of the Q-set and the interpretation of the data were researcher-led and thus open to researcher bias (Absalom-Hornby, Hare, Gooding, \& Tarrier, 2012). To limit this, each stage of the process involved more than one member of the research team and the Q-set was reviewed and refined with the assistance of subject specialists prior to being presented to participants. A further limitation of the study was the sample size which, while adequate for the analysis undertaken, was small and the practitioners were recruited from services that were similar in terms of the expectations of delivery. However, intervention training and the requirement for fidelity means that across different geographical and cultural contexts the expectations for the delivery of an intervention should be similar (Fixson et al., 2005) and practitioners will necessarily be faced with similar delivery challenges. Participants were also volunteers and they may have self-selected on the basis of their beliefs about PASS; however, it is likely that a study advertised as asking participants about their experiences of PASS would be as likely to draw in people with negative experiences as those with positive experiences. Finally, while this study is one of the first to enquire about practitioners' experiences and views of participating in supervision linked to the delivery of a PFS intervention, it is only able to 
provide data linked to one intervention. Further research is required about the experience of practitioners delivering other PFS interventions.

\section{Conclusions}

Although three factors, representing different viewpoints, were extracted from the data generated in this study, there is consistent evidence to suggest that PASS is an acceptable practitioner-focused intervention. Issues were raised about the feasibility of PASS but these do not appear to be detrimental to implementation. Rather they highlight the need for organizations to create an appropriate infrastructure to support supervision as a necessary priority for practitioners. Further details about the ways in which organizations can achieve appropriate infrastructural change to facilitate intervention success is currently emerging in the implementation science literature (e.g., Aarons et al., 2011; Schoenwald et al., 2013).

What remains unclear is the impact that participation in PASS has on intervention delivery and client outcomes. The three groups of practitioners offer different views on these relationships; however, it is essential that this is opened up to systematic testing. The findings from this study offer an opportunity to refine the PASS model prior to randomized controlled trial testing of the effectiveness of PASS, as compared to supervision as usual, as a method for changing practitioner consulting behavior, enhancing intervention delivery fidelity and improving client outcomes.

\section{Compliance with Ethical Standards}

\section{Disclosure of Potential Conflicts of Interest}

The Triple P - Positive Parenting Program is owned by The University of Queensland. The University, through its technology transfer company Uniquest Pty Ltd, has licensed Triple P International Pty Ltd to disseminate the program worldwide. Royalties stemming from this dissemination work are paid to UniQuest, which distributes payments to the University of Queensland Faculty of Health and Behavioural Sciences, School of Psychology, Parenting and Family Support Centre, and contributory authors in accordance with the

University's intellectual property policy. No author has any share or ownership in Triple $\mathrm{P}$ International. Matthew Sanders is the founder and lead author of the Triple P-Positive Parenting Program, and is a consultant to Triple P International. All other authors declare they have no conflict of interest. 
This study was funded by NHS Education Scotland.

\section{Research Involving Human Participants}

All procedures performed in this study involving human participants were in accordance with the ethical standards of, and approved by, Glasgow Caledonian University School of Health and Life Sciences Ethics Committee and were in accordance with the 1964 Helsinki declaration and its later amendments.

Informed Consent

Informed consent was obtained from all individuals participants included in the study. 


\section{References}

Aarons, G.A. (2004). Mental health provider attitudes toward adoption of evidence-based practice: The evidence-based practice attitude scale (EBPAS). Mental Health Services Research, 6, 6174

Aarons, G.A., Hurlburt, M., \& Horwitz, S.M. (2011). Advancing a conceptual model of evidence-based practice implementation in public service sectors. Adminitration and Policy in Mental Health Services Research, 38, 4-23.

Absalom-Hornby, V., Hare, D.J., Gooding, P., \& Tarrier, N. (2012). Attitudes of relatives and staff towards family intervention in forensic services using Q methodology. Journal of Psychiatric and Mental Health Nursing, 19, 162-173. doi:10.1111/j.1365-2850.2011.01770.x

Ayala, G.X., \& Elder, J.P. (2011). Qualitative methods to ensure acceptability of behavioral and social interventions to the target population. Journal of Public Health Dentistry, 71, S69-S79. doi:10.1111/j.1752-7325.2011.00241.x

Baker, R., Thompson, C., \& Mannion, R. (2006). Q methodology in health economics. Journal of Health Services Research \& Policy, 11, 38-45. doi:10.1258/135581906775094217

Berggren, I., Barbosa da Silva, A., \& Severinsson, E. (2005). Core ethical issues of clinical nursing supervision. Nursing \& Health Sciences, 7, 21-28. doi:10.1111/j.1442-2018.2005.00224.x

Breitenstein, S.M., \& Gross, D. (2013). Web-based delivery of a preventive parent training intervention: A feasibility study. Journal of Child and Adolescent Psychiatric Nursing, 26, 149157.

Brown, S.R. (1980). Political subjectivity, applications of Q methodology in political science. New Haven, Connecticut: Yale University Press.

Cheater, F.M., \& Hale, C. (2001). An evaluation of a local clinical supervision scheme for practice nurses. Journal of Clinical Nursing, 10, 119.

Counselman, E.F., \& Weber, R.L. (2004). Organizing and maintaining peer supervision groups. International Journal of Group Psychotherapy, 54, 125-143. doi:http://dx.doi.org/10.1521/ijgp.54.2.125.40391

Craig, P., Dieppe, P., Macintyre, S., Michie, S., Nazareth, I., \& Petticrew, M. (2013). Developing and evaluating complex interventions: The new Medical Research Council guidance. International Journal of Nursing Studies, 50, 587-592. doi:http://dx.doi.org/10.1016/.ijnurstu.2012.09.010 
Cross, R.M. (2005). Exploring attitudes: The case for Q methodology. Health Education Research, 20, 206-213. doi:10.1093/her/cyg121

Davy, J. (2002). Discursive reflections on a research agenda for clinical supervision. Psychology and Psychotherapy: Theory, Research and Practice, 75, 221-238. doi:http://dx.doi.org/10.1348/147608302169661

Fixsen, D.L., Blase, K.A., Duda, M.A., Naoom, S.F., \& Van Dyke, M. (2010). Implementation of evidence-based treatments for children and adolescents: Research findings and their implications for the future. In J. R. Weisz, \& A. E. Kazdin, Evidence-based psychotherapies for children and adolescents (2nd ed.) (pp. 435-450). New York, NY, US: Guilford Press, New York, NY.

Fixsen, D.L., Naoom, S.F., Blasé, K.A., Friedman, R.M. \& Wallace, F. (2005). Implementation research: A synthesis of the literature. Retrieved from http://ctndisseminationlibrary.org/PDF/nirnmonograph.pdf

Department of Health National Health Service Management Executive. (1993). A vision for the future: The nursing, midwifery and health visiting contribution to health and health care. London: Department of Health.

Haines, J., Mayorga, A.M., McDonald, J., O'Brien, A., Gross, D., Taveras, E.M., \& Gillman, M.W. (2012). Embedding weight-related messages within a general parenting programme: Development and feasibility evaluation of parents and tots together. Early Child Development and Care, 182, 951-965. doi:http://dx.doi.org/10.1080/03004430.2012.678592

Henggeler, S.W. (2011). Efficacy studies to large-scale transport: The development and validation of multisystemic therapy programs. Annual Review of Clinical Psychology, 7, 351-381. doi:http://dx.doi.org/10.1146/annurev-clinpsy-032210-104615

Karoly, P. (1993). Mechanisms of self-regulation: A systems view. Annual Review of Psychology, 44, 23-52. doi:http://dx.doi.org/10.1146/annurev.ps.44.020193.000323

Mazzucchelli, T., \& Sanders, M. (2010). Facilitating practitioner flexibility within an empirically supported intervention: Lessons from a system of parenting support. Clinical PsychologyScience and Practice, 17, 238-252. doi:10.1111/j.1468-2850.2010.01215.x

McKeown, B., \& Thomas, D. (1988). Q methodology. Thousand Oaks, CA, US: Sage Publications, Inc, Thousand Oaks, CA. 
McWilliam, J., Brown, J., Sanders, M.R.. \& Jones, L. (2015). The Triple P implementation framework: Enhancing outcomes from evidence-based programs. Manuscript in preparation.

Pitt, V.J., Lowe, D., Prictor, M., Hetrick, S., Ryan, R., Berends, L., \& Hill, S. (2013). A systematic review of consumer-providers' effects on client outcomes in statutory mental health services: The evidence and the path beyond. Journal of the Society for Social Work and Research, 4. doi:http://dx.doi.org/10.5243/jsswr.2013.21

Sanders, M.R., \& Murphy-Brennan, M. (2013). Triple P in action: Peer-assisted supervisions and support manual. Milton Australia: Triple P International Pty Ltd.

Sanders, M.R. (1999). Triple P-positive parenting program: Towards an empirically validated multilevel parenting and family support strategy for the prevention of behavior and emotional problems in children. Clinical Child and Family Psychology Review, 2, 71-90. doi:http://dx.doi.org/10.1023/A:1021843613840

Sanders, M.R. (2008). Triple P-positive parenting program as a public health approach to strengthening parenting. Journal of Family Psychology, 22, 506-517. doi:http://dx.doi.org/10.1037/0893-3200.22.3.506

Sanders, M.R. (2012). Development, evaluation, and multinational dissemination of the triple Ppositive parenting program. Annual Review of Clinical Psychology, 8, 345-379. doi:http://dx.doi.org/10.1146/annurev-clinpsy-032511-143104

Sanders, M.R., \& Murphy-Brennan, M. (2010). Creating conditions for success beyond the professional training environment. Clinical Psychology: Science and Practice, 17, 31-35. doi:http://dx.doi.org/10.1111/j.1468-2850.2009.01189.x

Sanders, M.R., Prinz, R.J., \& Shapiro, C.J. (2009). Predicting utilization of evidence-based parenting interventions with organizational, service-provider and client variables. Administration and Policy in Mental Health and Mental Health Services Research, 36, 133-143. doi:http://dx.doi.org/10.1007/s10488-009-0205-3

Schmolck, P. (n.d.) PQMethod manual. Retrieved from http://schmolck.userweb.mwn.de/qmethod/pqmanual.htm

Schoenwald, S.K., Megta, T.G., Frazier, S.L., \& Shernoff, E.S. (2013). Clinical supervision in effectiveness and implementation research. Clinical Psychology Science and Practice, 20, 4459. 
Schoenwald, S.K., Sheidow, A.J., \& Chapman, J.E. (2009). Clinical supervision in treatment transport: Effects on adherence and outcomes. Journal of Consulting and Clinical Psychology, 77, 410421. doi:http://dx.doi.org/10.1037/a0013788

Schoenwald, S.K., Sheidow, A.J., \& Letourneau, E.J. (2004). Toward effective quality assurance in evidence-based practice: Links between expert consultation, therapist fidelity, and child outcomes. Journal of Clinical Child and Adolescent Psychology, 33, 94-104. doi:http://dx.doi.org/10.1207/S15374424JCCP3301 10

Sexton, D., Snyder, P., Wadsworth, D., Jardine, A., \& James, E. (1998). Applying Q methodology to investigations of subjective judgements of early intervention effectiveness. Topics in Early Childhood Special Education, 18, 95.

Steckler, A., \& Linnan, L. (2003). Process evaluation for public health interventions and research. Portland: Ringgold Inc.

Stephenson, W. (1953). The study of behavior; Q-technique and its methodology. Chicago, IL, US: University of Chicago Press, Chicago, IL.

Tony, B., Louise, B., Christine, J., \& Majda, P. (2008). Wicked spell or magic bullet? A review of the clinical supervision literature 2001-2007. Nurse Education Today, 28, 264-272. doi:10.1016/j.nedt.2007.05.004

Turpin, G., \& Wheeler, S. (2011). IAPT supervision guidance. Retrieved from http://www.iapt.nhs.uk/silo/files/iapt-supervision-guidance-revised-march-2011.pdf

Ward, A. (2013). Self-regulatory peer supervision and support: An acceptability and feasibility study. Edinburgh: University of Edinburgh Masters Thesis.

Watkins C.E., \& Scaturo, D.J. (2013). Toward an integrative, learning-based model of psychtherapy supervision: Supervisory alliance, educational interventions, and supervisee learning/relearning. Journal of Psychotherapy Integration, 23, 75-95.

Watts, S., \& Stenner, P. (2012). Doing Q methodological research: Theory, method and interpretation. London: Sage Publications.

Wheeler, S., \& Richards, K. (2007). The impact of clinical supervision on counsellors and therapists, their practice and their clients. A systematic review of the literature. Counselling \& Psychotherapy Research, 7, 54-65. doi:http://dx.doi.org/10.1080/14733140601185274 